\title{
Energietechnik - aktuelle Anforderungen und Tendenzen
}

\author{
U. Schichler OVE
}

Online publiziert am 29. November 2017

(C) Springer-Verlag GmbH Austria, ein Teil von Springer Nature 2017

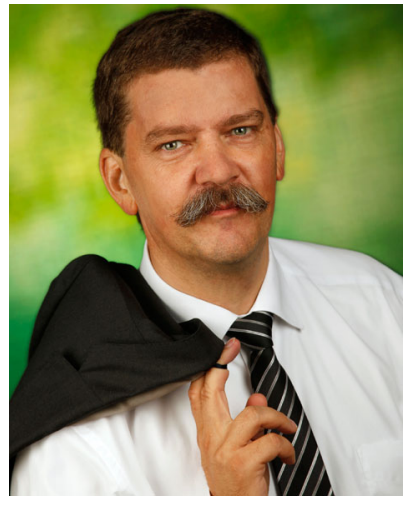

Univ.-Prof. Dr.-Ing. Uwe Schichler

währleisten Optimierungen ergeben sischer Energie zu geder Entwicklung neuer Isoliersysteme, dem Einsatz multiphysikalischer Simulationen, den zahlreichen Möglichkeiten der digitalen Mess- und Kommunikationstechnik und der Zustandsüberwachung von Betriebsmitteln. Die vorliegende e\&i-Ausgabe gibt einen ausgewählten Überblick zu den aktuellen Anforderungen und Tendenzen in der elektrischen Energietechnik.

Die erste Arbeit der vorliegenden Beiträge widmet sich dem Thema der Versorgungssicherheit und gibt ein aktuelles Lagebild aus der Sicht eines Übertragungsnetzbetreibers. Die nach dem Zweiten Weltkrieg aufgebauten Hoch- und Höchstspannungsnetze in der Übertragungsebene sind den heutigen Anforderungen im Hinblick auf die Netzstabilität, die stark volatilen Lastflüsse durch Veränderungen der Erzeugungs- und Lastschwerpunkte sowie die energiewirtschaftlichen Rahmenbedingungen teilweise nicht mehr gewachsen.

Der zweite Artikel beschäftigt sich mit der Zustandsbewertung rotierender elektrischer Maschinen auf der Basis von Teilentladungsmessungen. Neben einer Beschreibung der theoretischen Grundlagen zu den Teilentladungsquellen im elektrischen Isoliersystem rotierender Maschinen und des Ausbreitungsverhaltens von Teilentladungen in Maschinenwicklungen werden Messergebnisse vorgestellt und bewertet. Neuartige Verfahren zur Unterdrückung von Störsignalen und innovative Interpretationsmöglichkeiten sollen zukünftig eine automatische Analyse der Messergebnisse erlauben. Dadurch kann gegebenenfalls ein unvorhergesehener Ausfall des Betriebsmittels verhindert und die Versorgungssicherheit erhöht werden.

Im dritten Beitrag werden derzeit laufende Forschungsaktivitäten auf dem Gebiet der transienten Spannungen im österreichischen Hoch- und Höchstspannungsnetz in Folge von Blitzentladungen thematisiert. Neben einer Beschreibung der eingesetzten Messtechnik

${ }^{1}$ BRIC: Brasilien, Russland, Indien, China. werden ausgewählte Messergebnisse in Form von Fallbeispielen vorgestellt und analysiert. Darüber hinaus wird eine Korrelation der Messergebnisse mit den Daten des österreichischen Blitzortungssystems ALDIS durchgeführt.

Der vierte Artikel beschreibt aktuelle Forschungsergebnisse auf dem Gebiet der in den 1980er Jahren neu in den Hochspannungsnetzen eingeführten Metalloxid(MO)-Überspannungsableiter. Mit Hilfe von elektro-thermischen FEM-Simulationen werden die axiale Potential- und Temperaturverteilung von MO-Höchstspannungsableitern in Abhängigkeit verschiedener Steuerringkonfigurationen ermittelt. Es zeigt sich, dass der Grad der Feldsteuerung keinen Einfluss auf die thermische Stabilitätsgrenze hat und MOAbleiter auch bei einer nicht optimalen Potentialverteilung ordnungsgemäß funktionieren.

Der fünfte Beitrag beschäftigt sich mit schutztechnischen Maßnahmen für städtische Mittelspannungskabelnetze zur Verbesserung der Versorgungssicherheit. Mit Hilfe einer kurzzeitigen niederohmigen Phasen- bzw. Sternpunkterdung kann die aufgrund von einpoligen Kabelerdschlüssen auftretende Spannungsverlagerung in gelöscht betriebenen Mittelspannungsnetzen günstig beeinflusst werden. Es wird davon ausgegangen, dass es dadurch zu einer Verlängerung der Kabellebensdauer kommt. Die sich durch die erhöhten Fehlerströme in den Erdungssystemen ergebenden Fragen zur Personensicherheit werden umfangreich diskutiert.

Im sechsten Aufsatz setzt sich der Autor mit dem Einsatz und der Anwendung der Informations- und Kommunikationstechnik (IKT) im Bereich der Energieversorgungsunternehmen auseinander. Die IKT hat eine Schlüsselrolle im Bereich der durch verteilte Standorte und vernetzte Systeme bestimmten Energieversorgung und wird derzeit durch steigende Anforderungen in Form von großen Datenmengen (Smart Grids), Verfügbarkeit (Resilience) und Informationssicherheit (Internet of Things) geprägt. Dabei bekommt die IKT zukünftig insbesondere im Verteilnetz und bei der Entstehung von neuen Geschäftsmodellen im Bereich der Digitalisierung eine zunehmende Bedeutung.

Im abschließenden Bericht werden aktuelle Innovationen bei Transformatoren vorgestellt. Dabei stehen heute für die weltweit stetig steigenden technischen Anforderungen neben umweltfreundlichen Isolierflüssigkeiten anstelle von Mineralöl und berstsicheren Transformatorkesseln auch geräuschreduzierte Transformatorkonstruktionen zur Verfügung. Optimierte variable Drosselspulen und Phasenschiebertransformatoren stehen für eine gezielte Lastflusssteuerung im Übertragungsnetz zur Verfügung.

Ich bedanke mich bei allen Autoren für die Erstellung der Beiträge und bei den Reviewern sowie der Redaktion für die kritische und sorgfältige Durchsicht der Originalarbeiten. Ich wünsche Ihnen, liebe Leserinnen und Leser, eine interessante Lektüre der vorliegenden Beiträge.

Schichler, Uwe, Institut für Hochspannungstechnik und Systemmanagement, Technische Universität Graz, Inffeldgasse 18, 8010 Graz, Österreich (E-Mail: uwe.schichler@tugraz.at) 